Global COE Hi-Stat Discussion Paper Series 091

$$
\begin{gathered}
\text { Research Unit for Statistical } \\
\text { and Empirical Analysis in Social Sciences (Hi-Stat) }
\end{gathered}
$$

\title{
A Note on Utility Maximization with Unbounded Random Endowment
}

Keita Owari

October 2009 


\title{
A NOTE ON UTILITY MAXIMIZATION WITH UNBOUNDED RANDOM ENDOWMENT
}

\author{
KEITA OWARI \\ Graduate School of Economics, Hitotsubashi University \\ 2-1 Naka, Kunitachi, Tokyo 186-8601, Japan
}

\begin{abstract}
This paper addresses the applicability of the convex duality method for utility maximization, in the presence of random endowment. When the price process is a locally bounded semimartingale, we show that the fundamental duality relation holds true, for a wide class of utility functions and unbounded random endowments. We show this duality by exploiting Rockafellar's theorem on integral functionals, to a random utility function.
\end{abstract}

\section{INTRODUCTION}

Maximization of expected utility has been a time-honored issue in the study of mathematical finance. Especially, the following version of the problem with random endowment is important in view of its application to utility indifference valuation:

$$
\text { maximize } E\left[U\left(\theta \cdot S_{T}+B\right)\right], \quad \text { over all } \theta \in \Theta,
$$

where $U$ is an utility function, $S$ is a semimartingale, $\Theta$ is the set of admissible integrands (strategies), and $B$ is a random variable expressing a random endowment or a contingent claim.

A sophisticated way of solving (1.1) is the convex duality method which pass (1.1) to a minimization over the set of local martingale measures for $S$, through the (formal) duality equality:

$$
\sup _{\theta \in \Theta} E\left[U\left(\theta \cdot S_{T}+B\right)\right]=\inf _{\lambda>0} \inf _{Q \in \mathcal{M}} E\left[V\left(\lambda \frac{d Q}{d P}\right)+\lambda \frac{d Q}{d P} B\right],
$$

where $V$ is the Fenchel-Legendre transform of the utility function $U$, and $\mathcal{M}$ is a set of local martingale measures. The RHS of (1.2) is the optimal value of the dual problem. Note that the inequality " $\leq$ " is always true, while " $\geq$ " may not. This equality is shown by several authors in different settings, e.g., the case of no endowment $(B \equiv 0)$ by Kramkov and Schachermayer [12] and Schachermayer [17], the case of bounded $B$ by Bellini and Frittelli [2], and the case of exponential utility with suitably integrable $B$ by Delbaen et al. [5], Kabanov and Stricker [11] and Becherer [1].

Then a natural question arises: to what degree of generality does the equality (1.2) hold true ? This is the theme of this note. Under the fundamental assumption that $S$ is locally bounded, we shall prove the duality for a wide class of endowments $B$. Our idea is based on a refinement of [2] from a slightly different point of view. Namely, we view the problem

E-mail address: keita.owari@gmail.com, ed061002@g.hit-u.ac.jp.

2010 Mathematics Subject Classification. 91G80, 60H30, 46N10 .

Key words and phrases. Utility maximization, Convex duality method, Martingale measures. 
(1.1) as the maximization of expected utility functional associated to the random utility function $(\omega, x) \mapsto U(x+B(\omega))$. This allows us to take full advantage of Rockafellar's theorem on convex integral functionals.

\section{RESULT}

\subsection{SetuP}

Suppose we are given a complete probability space $(\Omega, \mathcal{F}, P)$ equipped with a filtration $\mathbb{F}:=\left(\mathcal{F}_{t}\right)_{t \in[0, T]}$ satisfying the usual conditions of right-continuity and completeness, where $T \in(0, \infty)$ is the fixed time horizon. We assume $\mathcal{F}=\mathcal{F}_{T}$ for notational simplicity. Let $S$ be a $d$-dimensional càdlàg locally bounded semimartingale on $\left(\Omega, \mathcal{F}_{T}, \mathbb{F}, P\right)$, and define

$$
\Theta_{b b}:=\left\{\theta \in L(S): \theta_{0}=0, \theta \cdot S \text { is uniformly bounded from below }\right\},
$$

where $L(S)=L(S, P)$ denotes the set of $d$-dimensional predictable processes $\theta=$ $\left(\theta^{1}, \ldots, \theta^{d}\right)$ which are $(S, P)$-integrable, and $\theta \cdot S=\int_{0}^{\cdot} \theta_{s} d S_{S}$ is the stochastic integral of $\theta \in L(S)$ w.r.t. $S$. For the precise definitions and basic properties of stochastic integrals and the set $L(S)$, we refer the reader to Jacod [9, 10]. Any $\theta \in \Theta_{b b}$ is called an admissible strategy, and we explicitly include the condition $\theta_{0}=0$ in the definition of admissibility to avoid the contribution of the initial value $\theta_{0} S_{0}$ to the stochastic integral.

In this paper, we consider only a class of utility functions defined on the whole real line. More precisely, we assume:

(A1) $U: \mathbb{R} \rightarrow \mathbb{R}$ is a continuously differentiable, increasing, and strictly concave function satisfying the so-called Inada condition:

$$
\lim _{x \rightarrow-\infty} U^{\prime}(x)=+\infty \text { and } \lim _{x \rightarrow+\infty} U^{\prime}(x)=0 .
$$

For a given utility function $U$, the Fenchel-Legendre transform of $U$ is defined by

$$
V(y):=\sup _{x \in \mathbb{R}}(U(x)-x y), \quad y \in \mathbb{R} .
$$

In the language of convex analysis, $V$ is the convex conjugate of the convex function $\Phi(x)=-U(-x)$. Under (A1), $V$ is also differentiable with $V^{\prime}(y)=-\left(U^{\prime}\right)^{-1}(y)$, and has the explicit representation: $V(y)=U\left(\left(U^{\prime}\right)^{-1}(y)\right)-y\left(U^{\prime}\right)^{-1}(y)$ if $y>0, V(0)=$ $U(+\infty):=\lim _{x \rightarrow+\infty} U(X)$, and $V(y)=+\infty$ if $y<0$. Furthermore, we have

$$
\lim _{y \downarrow 0} V^{\prime}(y)=-\infty \quad \text { and } \quad \lim _{y \rightarrow \infty} V^{\prime}(y)=+\infty .
$$

Note in particular that $V$ is bounded from below. For utility functions, we assume also the condition of reasonable asymptotic elasticities:

$$
A E_{-\infty}(U):=\liminf _{x \searrow-\infty} \frac{x U^{\prime}(x)}{U(x)}>1, \quad A E_{+\infty}(U):=\limsup _{x \nearrow+\infty} \frac{x U^{\prime}(x)}{U(x)}<1 .
$$

This condition is introduced by Kramkov and Schachermayer [12] and Schachermayer [17] as a necessary and sufficient condition for the existence of optimal investment strategy. Also, (A2) is equivalent to (see [6]): for any closed interval $[a, b] \subset(0, \infty)$, there exists $C_{1}, C_{2}>0$ such that

$$
V(\lambda y) \leq C_{1} V(y)+C_{2}(y+1), \quad \forall y>0, \lambda \in[a, b] .
$$

A probability measure $Q \ll P$ under which $S$ is a local martingale is called an absolutely continuous local martingale measure for $S$, and the set of all such measures is 
denoted by $\mathcal{M}_{\text {loc }}$. For the domain of the dual problem, we introduce the following subset of $\mathcal{M}_{\text {loc }}$ :

$$
\mathcal{M}_{V}:=\left\{Q \in \mathcal{M}_{\text {loc }}: E[V(d Q / d P)]<\infty\right\} .
$$

Note that, by the consequence (2.4) of (A2), we have for all $Q \ll P$,

$$
E[V(d Q / d P)]<\infty \quad \Leftrightarrow \quad E[V(\lambda d Q / d P)]<\infty, \quad \forall \lambda>0 .
$$

Generically, for any set $\mathcal{Q}$ of positive measures $Q \ll P$, we denote by $Q^{e}$ the set of $Q \in \mathcal{Q}$ with $Q \sim P$. We assume a version of no-arbitrage condition:

(A3) $\mathcal{M}_{V}^{e} \neq \emptyset$.

Finally, let $B$ be a $\mathcal{F}_{T}$-measurable random variable such that:

(A4) There exists some $\varepsilon>0$ for which,

$$
\begin{aligned}
& E\left[U\left(-(1+\varepsilon) B^{-}\right)\right]>-\infty, \\
& E\left[U\left(-\varepsilon B^{+}\right)\right]>-\infty
\end{aligned}
$$

\subsection{Main Theorem and Related Results}

We are now in the position to state the main theorem. The proof will be given in Section 3 .

Theorem 2.1. Under (A1)-(A4), the duality equality holds, i.e.,

$$
\sup _{\theta \in \Theta_{b b}} E\left[U\left(\theta \cdot S_{T}+B\right)\right]=\inf _{\lambda>0} \inf _{Q \in \mathcal{M}_{V}} E\left[V\left(\lambda \frac{d Q}{d P}\right)+\lambda \frac{d Q}{d P} B\right],
$$

and the infimum in the RHS is attained by some $(\hat{\lambda}, \hat{Q}) \in(0, \infty) \times \mathcal{M}_{V}^{e}$.

From a practical point of view, it is also important to ask whether the optimal expected utility can be approximated by bounded stochastic integrals, i.e., by admissible strategies such that $\theta \cdot S$ is bounded not only from below, but also from above. If the utility function is bounded from above, the answer is positive. Let

$$
\Theta_{b}=\left\{\theta \in L(S): \theta_{0}=0, \theta \cdot S \text { is uniformly bounded }\right\} .
$$

Corollary 2.2. If, in addition to (A1) - (A4), $U$ is bounded from above, then we have

$$
\sup _{\theta \in \Theta_{b}} E\left[U\left(\theta \cdot S_{T}+B\right)\right]=\inf _{\lambda>0} \inf _{Q \in \mathcal{M}_{V}} E\left[V\left(\lambda \frac{d Q}{d P}\right)+\lambda \frac{d Q}{d P} B\right] .
$$

Finally, as pointed out by [5] in the case of exponential utility, the duality equality is quite robust in the choice of admissible class. Let

$$
\Theta_{V}:=\left\{\theta \in L(S): \theta_{0}=0, \theta \cdot S \text { is a supermartingale under } \forall Q \in \mathcal{M}_{V}\right\} .
$$

Corollary 2.3. Suppose (A1) $-(A 4)$, and let $\Theta \subset L(S)$ be sandwiched by $\Theta_{b b}$ (resp. $\Theta_{b}$ if $U(\infty)<\infty$ ) and $\Theta_{V}$, i.e., $\Theta_{b b} \subset \Theta \subset \Theta_{V}$ (resp. $\Theta_{b} \subset \Theta \subset \Theta_{V}$ ). Then (2.7) remains true with $\Theta_{b b}$ replaced by $\Theta$.

We conclude this section with a brief review of related literature. Generally speaking, our result is an intermediate one among duality results of the type (1.2), in that, we require $S$ to be locally bounded, but give a duality of the classical-type (i.e., exclude the unpleasant intervention of bizarre singular term, see below) for a wide class of $U$ and $B$. 
Bounded Endowment. To our best knowledge, a duality result as our Theorem 2.1 appears first in [2]. Their argument (from our view point) is based on the analysis of the functional $X \mapsto E[U(X)]$ on $L^{\infty}$, and its conjugate defined on $b a \simeq\left(L^{\infty}\right)^{*}$ (Banach space of finitely additive signed measures), giving the duality for the case $B \equiv 0$. Then the case of bounded endowment follows by translation of the domain in $L^{\infty}$.

Exponential Utility. The "Six-Author Paper" [5] and its refinement [11] develop a general duality theory for the case of exponential utility: $U(x)=1-e^{-\alpha x}$, giving the duality equality under (2.5) and the boundedness from above of $B$. This assumption is weakened by [1] to the condition corresponding to our (A4). More recently, Owari [13] extends this framework to the robust exponential utility maximization.

General Semimartingales. Without doubt, the duality theory can be extended to the case with non-locally bounded $S$. In this case, however, the duality equality holds only in a generalized sense as (Biagini et al. [3]):

$$
\sup _{\theta \in \mathcal{H}^{W}} E\left[U\left(\theta \cdot S_{T}+B\right)\right]=\inf _{\lambda>0} \inf _{Q \in \mathcal{M}^{W}}\left(E\left[V\left(\lambda \frac{d Q^{r}}{d P}\right)\right]+\lambda Q(B)+\lambda\left\|Q^{s}\right\|\right) .
$$

where $\mathcal{H}^{W}$ is the set of integrands of which $\theta \cdot S$ is bounded from below by a suitable random variable $W, \mathcal{M}^{W}$ is a subset of $b a, Q(B)$ is the "integral" of $B$ w.r.t. a finitely additive measure $Q$, and $Q^{r}$ (resp. $Q^{s}$ ) denotes the regular (resp. singular) part of $Q$ in the Hewitt-Yosida decomposition. Our integrability assumption (A4) appears in [3]. In this respect, Theorem 2.1 states that, in the case of locally bounded $S$, the singular term automatically disappears, whenever $B$ satisfies (A4), although the case where $B$ satisfies (2.5) and (2.6) for " $\forall \varepsilon>0$ " is covered by [3].

Other Case. Yet another approach is proposed by [14]. There the problem (1.1) is considered under the assumption that there exists $x^{\prime}, x^{\prime \prime} \in \mathbb{R}$ and $\theta^{\prime}, \theta^{\prime \prime} \in \Theta_{V}$ such that

$$
x^{\prime}+\theta^{\prime} \cdot S_{T} \leq B \leq x^{\prime \prime}+\theta^{\prime \prime} \cdot S_{T},
$$

and $\theta^{\prime} \cdot S$ is a martingale under every $Q \in \mathcal{M}_{V}$. This has no apparent relation to our assumption. In contrast to this formulation, our approach has an advantage that we need only the integrability conditions for $B$, which are easily checked $a$ priori, while (2.11) is hard to verify.

Remark 2.4. Since we focus only on the case of utility on $\mathbb{R}$, articles on the case of utility on $\mathbb{R}_{+}$are omitted. For this direction, see e.g., Cvitanić et al. [4], Hugonnier and Kramkov [7], Hugonnier et al. [8] and references therein.

\section{PROOFS}

\subsection{OutLine}

We first give the outline of the proof, which may help the understanding. Roughly speaking, our idea is based on Bellini and Frittelli [2], but exploits Rockafellar's theorem [15] on convex integral functionals to a random utility function.

As most of literature on this subject, we first reduce the problem to a maximization of a concave functional defined on $L^{\infty}$, and then appeal to the $\left(L^{\infty}, b a\right)$-duality. Define

$$
\mathcal{C}:=\left\{X \in L^{\infty}: \exists \theta \in \Theta_{b b} \text { such that } X \leq \theta \cdot S_{T}\right\},
$$

which is a convex cone containing $L_{-}^{\infty}$ and $\mathcal{K}:=\left\{\theta \cdot S_{T}: \theta \in \Theta_{b}\right\}$ (see e.g., [2]). As in [2], we can show (Lemma 3.6 below):

$$
\sup _{\Theta_{b b}} E\left[U\left(\theta \cdot S_{T}+B\right)\right]=\sup _{X \in \mathcal{C}} E[U(X+B)] .
$$


Let $\delta_{\mathcal{C}}(X)=0$ if $X \in \mathcal{C}$ and $=+\infty$ otherwise (i.e., $\delta_{\mathcal{C}}$ is the indicator function of $\mathcal{C}$ in the sense of convex analysis), and define (formally) a concave functional $u_{B}$ on $L^{\infty}$ by

$$
u_{B}(X):=E[U(X+B)] .
$$

Then we have

$$
\sup _{X \in \mathcal{C}} u_{B}(X)=\sup _{X \in L^{\infty}}\left(u_{B}(X)-\delta_{\mathcal{C}}(X)\right),
$$

Now if $u_{B}$ is well-defined and regular enough, Fenchel's duality theorem shows that

$$
\sup _{X \in L^{\infty}}\left(u_{B}(X)-\delta_{\mathcal{C}}(X)\right)=\min _{v \in b a}\left(\delta_{\mathcal{C}}^{*}(v)-u_{B}^{*}(v)\right)=\min _{v \in b a}\left(v_{B}(v)+\delta_{\mathcal{C}}^{*}(v)\right),
$$

where $v_{B}$ is the conjugate of $u_{B}$ defined on $b a$ by

$$
v_{B}(v):=\sup _{X \in L^{\infty}}\left(u_{B}(X)-v(X)\right), \quad v \in b a .
$$

Thus, the key step is to verify the regularity of $u_{B}$ and to derive the explicit form of $v_{B}$. We will do this (Proposition 3.8) by exploiting Rockafellar's theorem to $u_{B}$ which is a concave integral functional defined by the random concave function $U_{B}$ on $\Omega \times \mathbb{R}: U_{B}(\omega, x):=$ $U(x+B(\omega))$. In this step, the assumption (A4) plays a crucial role, giving the estimates between $U, U_{B}$ and $V$ (Lemma 3.4).

\subsection{Preliminaries And Important Estimates}

We first introduce some additional notations and concepts used in the proof of Theorem 2.1. The first one is the description of the space $b a$.

Definition 3.1 $\left(b a\left(\Omega, \mathcal{F}_{T}, P\right)\right)$. $\quad b a:=b a\left(\Omega, \mathcal{F}_{T}, P\right)$ is the set of all bounded finitely additive measures absolutely continuous w.r.t. $P$, i.e., $v \in b a\left(\Omega, \mathcal{F}_{T}, P\right)$ if and only if $v$ is a real valued function on $\mathcal{F}_{T}$ such that (1) $\sup _{A \in \mathcal{F}_{T}}|\nu(A)|<\infty$, (2) for every $A \in \mathcal{F}_{T}$, $P(A)=0$ implies $v(A)=0$, (3) if $A, B \in \mathcal{F}_{T}$ and $A \cap B=\emptyset$, then $v(A \cup B)=$ $v(A)+v(B)$. Also, $b a_{+}$(resp. $\left.b a^{\sigma}\right)$ denotes the set of positive (resp. $\sigma$-additive) elements of $b a$, and set $b a_{+}^{\sigma}:=b a_{+} \cap b a^{\sigma}, b a_{+}^{\sigma, 1}:=\left\{v \in b a_{+}^{\sigma}: v(\Omega)=1\right\}$, and

$$
\mathcal{Q}_{V}:=\left\{v \in b a_{+}^{\sigma}: E[V(d v / d P)]<\infty\right\} .
$$

Only facts which will be used here are: (1) $b a$ is a Banach space equipped with the total variation norm, and $b a \simeq\left(L^{\infty}\right)^{*}$, (2) every $v \in b a$ has a unique decomposition $v=v^{r}+v^{s}$, where $v^{r} \in b a^{\sigma}$ and $v^{s}$ is purely finitely additive. $b a_{+}^{\sigma, 1}$ is nothing but the set of probabilities $Q$ on $\left(\Omega, \mathcal{F}_{T}\right)$ with $Q \ll P$. Also, as a direct consequence of (2.4), $\mathcal{Q}_{V}$ is a convex cone having the following representation:

\section{Lemma 3.2.}

1. If $V(0)=U(\infty)<\infty$,

$$
\mathcal{Q}_{V}=\left\{\lambda Q: \lambda \geq 0, Q \in b a_{+}^{\sigma, 1}, E[V(d Q / d P)]<\infty\right\} .
$$

2. If $V(0)=+\infty$,

$$
\mathcal{Q}_{V}=\left\{\lambda Q: \lambda>0, Q \in b a_{+}^{\sigma, 1}, E[V(d Q / d P)]<\infty\right\} .
$$

Recall that the set $\mathcal{C}$ (defined by (3.1)) is a convex cone containing $L_{-}^{\infty}$. The following relation between $\mathcal{C}$ and $\mathcal{M}_{\text {loc }}$ is well-known (e.g., [2, Lemma 1.1]): for every $Q \in b a_{+}^{\sigma, 1}$,

$$
Q \in \mathcal{M}_{\text {loc }} \Leftrightarrow E^{Q}[X] \leq 0, \text { for } \forall X \in \mathcal{C} .
$$


Let $\delta_{\mathcal{C}}^{*}$ be the conjugate of the indicator function $\delta_{\mathcal{C}}$, i.e.,

$$
\delta_{\mathcal{C}}^{*}(v)=\sup _{X \in L^{\infty}}\left(v(X)-\delta_{\mathcal{C}}(X)\right)=\sup _{X \in \mathcal{C}} v(X), \quad \forall v \in b a .
$$

The above observations immediately yield the next lemma.

Lemma 3.3. $\delta_{\mathcal{C}}^{*}(v)=+\infty$ if $v \notin b a_{+}$, and for all $v \in b a_{+}^{\sigma}$,

$$
\delta_{\mathcal{C}}^{*}(v)= \begin{cases}0 & \text { if } v \in \operatorname{cone}\left(\mathcal{M}_{\text {loc }}\right) \\ +\infty & \text { otherwise. }\end{cases}
$$

Here

$$
\operatorname{cone}\left(\mathcal{M}_{\text {loc }}\right)=\left\{\lambda Q: \lambda \geq 0, Q \in \mathcal{M}_{\text {loc }}\right\}
$$

Proof. If $v \notin b a$, there exists $\bar{X} \in L_{+}^{\infty}$ with $v(\bar{X})<0$. Since $L_{-}^{\infty} \subset \mathcal{C}$, we have $-\lambda \bar{X} \in \mathcal{C}$ for all $\lambda>0$, hence

$$
\delta_{\mathcal{C}}^{*}(v) \geq \sup _{\lambda>0}-\lambda v(\bar{X})=+\infty .
$$

The fact that $\mathcal{C}$ is a cone implies that $\delta_{\mathcal{C}}^{*}$ is $\{0,+\infty\}$-valued, and $\delta_{\mathcal{C}}^{*}(\nu)=0$ if and only if $v(X) \leq 0$ for all $X \in \mathcal{C}$. If $v \in b a_{+}^{\sigma}$, the latter condition is equivalent to saying that $v \in \operatorname{cone}\left(\mathcal{M}_{\text {loc }}\right)$ by (3.6).

The following estimates are elementary, but play a key role in the proof of theorem.

Lemma 3.4. Let $\varepsilon>0$.

(a) For every random variable $Y \geq 0$,

$$
\begin{aligned}
\frac{\varepsilon}{1+\varepsilon}(V(Y)-V(1))+U\left(-(1+\varepsilon) B^{-}\right) & \leq V(Y)+Y B \\
& \leq \frac{1+\varepsilon}{\varepsilon} V(Y)-\frac{1}{\varepsilon} U\left(-\varepsilon B^{+}\right) .
\end{aligned}
$$

(b) For every $Y \geq 0$ and every random variable $X$,

$$
\begin{aligned}
& \frac{\varepsilon}{1+\varepsilon} U\left(\frac{1+\varepsilon}{\varepsilon} X\right)+\frac{1}{1+\varepsilon} U\left(-(1+\varepsilon) B^{-}\right) \leq U(X+B) \\
& \leq \frac{1+\varepsilon}{\varepsilon} V(Y)+X Y-\frac{1}{\varepsilon} U\left(-\varepsilon B^{+}\right) .
\end{aligned}
$$

Remark 3.5. We make some remarks on the consequences of (A4).

1. (3.8) implies that $V(Y) \in L^{1}$ if and only if $V(Y)+Y B \in L^{1}$, and in this case, $Y B \in L^{1}$ and $E[V(Y)+Y B]=E[V(Y)]+E[Y B]$. In particular, for any $Q \in b a_{+}^{\sigma, 1}$, $E[V(d Q / d P)]<\infty$ implies $B \in L^{1}(Q)$.

2. The map $(\lambda, Q) \mapsto E[V(\lambda d Q / d P)+\lambda(d Q / d P) B]$ on $\mathbb{R}_{+} \times b a_{+}^{\sigma, 1}$ to $(-\infty,+\infty]$ is well-defined (note that $V$ is bounded from below), and is finite if and only if $\lambda Q \in \mathcal{Q}_{V}$. Let $\lambda, Q$ be such a pair. Then by Jensen's inequality,

$$
E\left[V\left(\lambda \frac{d Q}{d P}\right)+\lambda \frac{d Q}{d P} B\right] \geq \frac{\varepsilon}{1+\varepsilon}(V(\lambda)-V(1))+E\left[U\left(-(1+\varepsilon) B^{-}\right)\right] .
$$

In particular, $\inf _{\lambda \geq 0, Q \in \mathcal{M}_{V}} E[V(\lambda d Q / d P)+\lambda(d Q / d P) B]>-\infty$, since again $V$ is bounded from below.

3. (A3) and (A4) implies that $U(X+B) \in L^{1}$ for every $X \in L^{\infty}$. Indeed, the LHS of (3.9) is integrable for any $X \in L^{\infty}$ since $U$ is monotone, while the RHS is integrable for $Y=d \bar{Q} / d P$ with $\bar{Q} \in \mathcal{M}_{V}$. 
Proof of Lemma. (a) For any $Y \geq 0$,

$$
\varepsilon Y B \leq Y\left(\varepsilon B^{+}\right) \leq V(Y)-U\left(-\varepsilon B^{+}\right),
$$

by Young's inequality, thus,

$$
V(Y)+Y B \leq \frac{1+\varepsilon}{\varepsilon} V(Y)-\frac{1}{\varepsilon} U\left(-\varepsilon B^{+}\right),
$$

and we get the second inequality in (3.8). On the other hand,

$$
\begin{aligned}
Y B^{-} & =\frac{Y}{1+\varepsilon}(1+\varepsilon) B^{-} \leq\left(\frac{\varepsilon}{1+\varepsilon}+\frac{1}{1+\varepsilon} Y\right)(1+\varepsilon) B^{-} \\
& \leq V\left(\frac{\varepsilon}{1+\varepsilon}+\frac{1}{1+\varepsilon} Y\right)-U\left(-(1+\varepsilon) B^{-}\right) \\
& \leq \frac{1}{1+\varepsilon} V(Y)+\frac{\varepsilon}{1+\varepsilon} V(1)-U\left(-(1+\varepsilon) B^{-}\right) .
\end{aligned}
$$

Using this,

$$
V(Y)+Y B \geq V(Y)-Y B^{-} \geq \frac{\varepsilon}{1+\varepsilon} V(Y)-\frac{\varepsilon}{1+\varepsilon} V(1)+U\left(-(1+\varepsilon) B^{-}\right) .
$$

These prove the assertion (a).

(b) For any random variable $X$ and positive random variable $Y$,

$$
\begin{aligned}
U(X+B) & \leq V(Y)+Y(X+B) \\
& \leq \frac{1+\varepsilon}{\varepsilon} V(Y)+X Y-\frac{1}{\varepsilon} U\left(-\varepsilon B^{+}\right),
\end{aligned}
$$

by (3.11). Also, since $U$ is concave and monotone increasing,

$$
\begin{aligned}
U(X+B) & =U\left(\frac{\varepsilon}{1+\varepsilon} \cdot \frac{1+\varepsilon}{\varepsilon} X+\frac{1}{1+\varepsilon} \cdot(1+\varepsilon) B\right) \\
& \geq \frac{\varepsilon}{1+\varepsilon} U\left(\frac{1+\varepsilon}{\varepsilon} X\right)+\frac{1}{1+\varepsilon} U((1+\varepsilon) B) \\
& \geq \frac{\varepsilon}{1+\varepsilon} U\left(\frac{1+\varepsilon}{\varepsilon} X\right)+\frac{1}{1+\varepsilon} U\left(-(1+\varepsilon) B^{-}\right) .
\end{aligned}
$$

This completes the proof.

We now reduce the problem to a minimization in $\mathcal{C}$.

Lemma 3.6. We have

$$
\sup _{\theta \in \Theta_{b b}} E\left[U\left(\theta \cdot S_{T}+B\right)\right]=\sup _{X \in \mathcal{C}} E[U(X+B)] .
$$

Proof. The inequality " $\geq$ " is immediate from the definition of $\mathcal{C}$ and the monotonicity of $U$. Let $\theta \in \Theta_{b b}$. Then for any $k \in \mathbb{N}, X_{k}:=\left(\theta \cdot S_{T}\right) \wedge k$ is in $\mathcal{C}$. Since $\theta \in \Theta_{b b}$, there exists $x>0$ with $\theta \cdot S \geq-x$ uniformly, a.s., hence $X_{k} \geq-x$, a.s. We have

$$
U\left(X_{k}+B\right) \nearrow U\left(\theta \cdot S_{T}+B\right), \quad \text { a.s. }
$$

Now Lemma 3.4 (b) implies that $U\left(X_{k}+B\right) \geq \frac{\varepsilon}{1+\varepsilon} U\left(\frac{-(1+\varepsilon)}{\varepsilon} x\right)+\frac{1}{1+\varepsilon} U\left(-(1+\varepsilon) B^{-}\right)$, for each $k$, which is in $L^{1}$ by (A4). On the other hand, taking $Q \in \mathcal{M}_{V}$ (by (A3)), $U\left(\theta \cdot S_{T}+B\right) \leq \frac{1+\varepsilon}{\varepsilon} V(d Q / d P)+\theta \cdot S_{T} d Q / d P-\frac{1}{\varepsilon} U\left(-\varepsilon B^{+}\right) \in L^{1}$, since $\theta \cdot S$ is a $Q$-supermartingale, and $U\left(-\varepsilon B^{+}\right) \in L^{1}$ by (A4). Therefore, the convergence (3.13) takes place in $L^{1}$ by the dominated convergence theorem, hence $\lim _{k \rightarrow \infty} E\left[U\left(X_{k}+B\right)\right]=$ $E\left[U\left(\theta \cdot S_{T}+B\right)\right]$. This proves the inequality " $\leq$ ". 
The final lemma in this subsection states that the infimum in the dual problem must not attained neither by $\lambda=0$ nor by $Q \chi P$.

Lemma 3.7. If $v \in \mathcal{Q}_{V} \backslash \mathcal{Q}_{V}^{e}$, there exists $\tilde{v} \in \mathcal{Q}_{V}^{e}$ such that

$$
E\left[V\left(\frac{d \tilde{v}}{d P}\right)+\frac{d \tilde{v}}{d P} B\right]<E\left[V\left(\frac{d v}{d P}\right)+\frac{d v}{d P} B\right] .
$$

Proof. This is trivial if $V(0)=+\infty$ since then $\mathcal{Q}_{V}=\mathcal{Q}_{V}^{e}$, thus we assume $V(0)<\infty$. Let $v \in \mathcal{Q}_{V}$ and $\bar{v} \in \mathcal{Q}_{V}^{e}\left(\neq \emptyset\right.$ by (A3)). Set $v_{\alpha}:=\alpha \bar{v}+(1-\alpha) v \in \mathcal{Q}_{V}(\alpha \in[0,1])$. Note that $v_{\alpha} \in \mathcal{Q}_{V}^{e}$ for $\alpha \in(0,1)$. Set also,

$$
\varphi_{\alpha}:=V\left(\frac{d v_{\alpha}}{d P}\right)+\frac{d v_{\alpha}}{d P} B \in L^{1}
$$

Since $\alpha \mapsto \varphi_{\alpha}(\omega)$ is convex for a.e. $\omega, \alpha \mapsto\left(\varphi_{\alpha}-\varphi_{0}\right) / \alpha$ is increasing in $\alpha$, hence

$$
\frac{\varphi_{\alpha}-\varphi_{0}}{\alpha} \searrow Z, \quad \text { a.s., }
$$

for some random variable $Z$. Since $\left(\varphi_{1}-\varphi_{0}\right) / \alpha \in L^{1}$, we can apply the monotone convergence theorem to get

$$
\lim _{\alpha \searrow 0} E\left[\frac{\varphi_{\alpha}-\varphi_{0}}{\alpha}\right]=E[Z]
$$

On the other hand,

$$
Z=\left(V^{\prime}\left(\frac{d \nu}{d P}\right)+B\right)\left(\frac{d \bar{\nu}}{d P}-\frac{d \nu}{d P}\right)=-\infty \text { on }\left\{\frac{d \nu}{d P}=0\right\},
$$

since $V^{\prime}(0)=-\infty$ (by (A1)) and $\bar{v} \in \mathcal{Q}_{V}^{e}$. Therefore, (3.14) shows that if $v \not P$, there exists $\alpha \in(0,1)$ such that

$$
E\left[V\left(\frac{d v_{\alpha}}{d P}\right)+\frac{d v_{\alpha}}{d P} B\right]-E\left[V\left(\frac{d v}{d P}\right)+\frac{d v}{d P} B\right]<-\alpha .
$$

Since $v_{\alpha} \in \mathcal{Q}_{V}^{e}$, we have the desired result.

\subsection{Description of The Conjugate Functional}

We now come to the key step, namely, the regularity of $u_{B}$ defined by (3.2), and the description of its conjugate $v_{B}$ defined by (3.3).

Proposition 3.8. Assume $(A 1)-(A 4)$. Then

(a) $u_{B}$ is well-defined and continuous on $L^{\infty}$ w.r.t. the norm topology.

(b) $v_{B}$ has the expression:

$$
v_{B}(v)= \begin{cases}E\left[V\left(\frac{d v}{d P}\right)+\frac{d v}{d P} B\right] & \text { if } v \in \mathcal{Q}_{V} \\ +\infty & \text { otherwise }\end{cases}
$$

We shall prove this by exploiting Rockafellar's theorem on convex integral functionals. We begin with some preparation.

Definition 3.9. A map $f: \Omega \times \mathbb{R} \rightarrow \mathbb{R} \cup\{+\infty\}$ is called a normal convex integrand if:

(a) $f$ is jointly measurable (i.e., $\mathcal{F} \times \mathcal{B}(\mathbb{R})$-measurable),

(b) $x \mapsto f(\omega, x)$ is a lower semicontinuous proper convex function for a.e. $\omega$.

Also, the conjugate random convex function of $f$ is defined by

$$
f^{*}(\omega, y):=\sup _{x \in \mathbb{R}}(x y-f(\omega, x)), \quad(\omega, y) \in \Omega \times \mathbb{R} .
$$


We cite here Rockafellar's theorem in a form suited to our purpose.

Theorem 3.10 (Rockafellar [15], Theorem 1, Corollary 2A).

1. Let $f: \Omega \times \mathbb{R} \rightarrow \mathbb{R}$ be a random convex function such that

(a) there exists some $X \in L^{\infty}$ such that, $f(\cdot, X(\cdot))^{+} \in L^{1}$,

(b) there exists some $Y \in L^{1}$ such that, $f^{*}(\cdot Y(\cdot))^{+} \in L^{1}$.

Then the map

$$
I_{f}(X):=E[f(X)]=\int_{\Omega} f(\omega, X(\omega)) P(d \omega), \quad X \in L^{\infty}
$$

is well-defined as a convex functional on $L^{\infty}$, and the conjugate $I_{f}^{*}: b a \mapsto \mathbb{R} \cup\{+\infty\}$ is expressed as:

$$
I_{f}^{*}(v)=I_{f}^{*}\left(v^{r}\right)+\delta_{\operatorname{dom}\left(I_{f}\right)}^{*}\left(v^{s}\right), \quad v \in b a,
$$

where,

$$
\begin{aligned}
I_{f^{*}}\left(v^{r}\right) & =E\left[f^{*}\left(d v^{r} / d P\right)\right]=\int_{\Omega} f^{*}\left(\omega, \frac{d v^{r}}{d P}(\omega)\right) P(d \omega), \\
\delta_{\operatorname{dom}\left(I_{f}\right)}^{*}\left(v^{s}\right) & =\sup _{X \in \operatorname{dom}\left(I_{f}\right)} v^{s}(X) .
\end{aligned}
$$

2. If in addition $f(\cdot, X(\cdot)) \in L^{1}$ for every $X \in L^{\infty}$, then $I_{f}$ is continuous on $L^{\infty}$ and

$$
I_{f}^{*}(v)= \begin{cases}E\left[f^{*}(d v / d P)\right] & \text { if } v \in b a^{\sigma} \\ +\infty & \text { otherwise. }\end{cases}
$$

Remark 3.11. In [15], the notion of normal convex integrands is introduced in a slightly different way, which is equivalent to our Definition 3.9 if the underlying probability space is complete as we assumed. See Rockafellar and Wets [16], Ch.14 for detail. Also, the original version of Theorem 3.10 in [15] is stated and proved on a $\sigma$-finite measure space, rather than a probability space.

Proof of Proposition 3.8. We apply Rockafellar's theorem to the random convex function

$$
f(\omega, x)=-U(-x+B(\omega)),
$$

which is clearly jointly measurable, convex and continuous in $x$, hence normal. The conjugate $f^{*}$ is given by

$$
f^{*}(\omega, y)=V(y)+y B(\omega),
$$

and $I_{f}(X)=E[-U(-X+B)]=-u_{B}(-X)$, thus $I_{f}^{*}=v_{B}$.

For every $X \in L^{\infty}, f(X)=-U(-X+B)$ is integrable by Lemma 3.4 and Remark 3.5. On the other hand, we can take $\bar{Q} \in \mathcal{M}_{V}$ by (A3), so that $f^{*}(d \bar{Q} / d P)=V(d \bar{Q} / d P)+$ $(d \bar{Q} / d P) B \in L^{1}$, by Lemma 3.4. Hence we can apply Theorem 3.10 to get the assertion (a), and that

$$
v_{B}(v)=I_{f}^{*}(v)= \begin{cases}E\left[V\left(\frac{d v}{d P}\right)+\frac{d v}{d P} B\right] & \text { if } v \in b a^{\sigma} \\ +\infty & \text { otherwise. }\end{cases}
$$

It remains to show that $v_{B}(v)=+\infty$ if $v \in b a^{\sigma} \backslash \mathcal{Q}_{V}$. Suppose $v \in b a^{\sigma} \backslash b a_{+}^{\sigma}$. Since $f^{*}(d v / d P)=V(d v / d P)+(d v / d P) B=+\infty$ on the set $\{d v / d P<0\}$ which has a positive probability, the estimate (3.8) of Lemma 3.4 shows that $v_{B}(v)=E\left[f^{*}(d v / d P)\right]=$ $+\infty$. Finally, for any $Y \geq 0 f^{*}(Y) \in L^{1}$ if and only if $V(Y) \in L^{1}$ by Remark 3.5, hence $v_{B}(v)<\infty$ if and only if $v \in \mathcal{Q}_{V}$. 


\subsection{Proof of Main Results}

Proof of Theorem 2.1. We apply Fenchel's theorem for $\left(L^{\infty}, b a\right)$ to $u_{B}$ and $\delta_{\mathcal{C}}$. By Proposition $3.8, \operatorname{dom}\left(u_{B}\right)=L^{\infty}$ and $u_{B}$ is continuous, hence epi $\left(u_{B}\right)$ has non-empty interior w.r.t. the product topology of $L^{\infty} \times \mathbb{R}$. Indeed, $\left(0, u_{B}(0)-1\right)$ is an interior point of epi $\left(u_{B}\right)$. Also, $\operatorname{dom}\left(u_{B}\right) \cap \operatorname{dom}\left(\delta_{\mathcal{C}}\right)=\mathcal{C}$ has an interior point, since $L_{-}^{\infty} \subset \mathcal{C}$, and $X \equiv-1$ is an interior point of $L_{-}^{\infty}$. Using (3.6), Lemma 3.4, and (A4),

$$
\begin{aligned}
\sup _{X \in L^{\infty}}\left(u_{B}(X)-\delta_{\mathcal{C}}(X)\right) & =\sup _{X \in \mathcal{C}} E[U(X+B)] \\
& \leq \frac{1+\varepsilon}{\varepsilon} E\left[V\left(\frac{d \bar{Q}}{d P}\right)\right]-\frac{1}{\varepsilon} E\left[U\left(-\varepsilon B^{+}\right)\right]+\sup _{X \in \mathcal{C}} E^{\bar{Q}}[X] \\
& \leq \frac{1+\varepsilon}{\varepsilon} E\left[V\left(\frac{d \bar{Q}}{d P}\right)\right]-\frac{1}{\varepsilon} E\left[U\left(-\varepsilon B^{+}\right)\right]<\infty .
\end{aligned}
$$

where $\bar{Q}$ is an element of $\mathcal{M}_{V}(\neq \emptyset$ by (A3)). Therefore, we can apply Fenchel's theorem to get

$$
\begin{aligned}
\sup _{X \in C} u_{B}(X) & =\sup _{X \in L^{\infty}}\left(u_{B}(X)-\delta_{C}(X)\right)=\min _{v \in b a}\left(v_{B}(v)+\delta_{C}^{*}(v)\right) \\
& =\min _{v \in \mathcal{Q}_{V}}\left(E\left[V\left(\frac{d v}{d P}\right)+\frac{d v}{d P} B\right]+\delta_{C}^{*}(v)\right) \\
& =\min _{\lambda>0, Q \in \mathcal{M}_{V}^{e}} E\left[V\left(\lambda \frac{d Q}{d P}\right)+\lambda \frac{d Q}{d P}\right] .
\end{aligned}
$$

Here, the third equality follows from Proposition 3.8, and the fourth from Lemma 3.3 and Lemma 3.7. Now Theorem 2.1 follows from Lemma 3.6.

Proof of Corollary 2.2. This is a direct consequence of the following minor modification of Kabanov and Stricker [11], Lemma 5.1.

Lemma 3.12. Suppose that $U$ is bounded from above. Then for any $\theta \in \Theta_{b b}$, there exists a sequence $\left(\theta^{n}\right) \subset \Theta_{b}$ such that $\left(\left(\theta-\theta^{n}\right) \cdot S\right)_{T}^{*} \rightarrow 0$ in probability and

$$
E\left[U\left(\theta \cdot S_{T}+B\right)\right]=\lim _{n \rightarrow \infty} E\left[U\left(\theta^{n} \cdot S_{T}+B\right)\right]
$$

Proof. Since $S$ is locally bounded, we can take a increasing sequence $\left(\tau_{n}\right)_{n}$ of stopping times with $S_{\tau_{n}}^{*} \leq n$, and $\tau_{n} \nearrow T$, stationarily, a.s. Then $\left(\left(\theta 1_{\llbracket 0, \tau_{n} \rrbracket}-\theta\right) \cdot S\right)_{T}^{*} \rightarrow 0$ in probability, for any $\theta \in L(S)$. Thus, if $\theta \cdot S \geq-x$, we have $U\left(\theta \cdot S_{T}^{\tau_{n}}+B\right) \rightarrow$ $U\left(\theta \cdot S_{T}+B\right)$ in probability, and this sequence is uniformly bounded from below (resp. above) by $\frac{\varepsilon}{1+\varepsilon} U\left(\frac{-(\varepsilon+1)}{\varepsilon} x\right)+\frac{1}{1+\varepsilon} U\left(-(1+\varepsilon) B^{-}\right) \in L^{1}$ (resp. $\left.U(\infty)<\infty\right)$ by Lemma 3.4 (b) and (A4). Hence the dominated convergence theorem shows that

$$
\lim _{n \rightarrow \infty} E\left[U\left(\theta \cdot S_{T}^{\tau_{n}}+B\right)\right]=E\left[U\left(\theta \cdot S_{T}+B\right)\right]
$$

This reduces the assertion to the case where $S$ is uniformly bounded by some constant $c$.

Suppose that $\theta \cdot S$ is uniformly bounded from below by $a>0$. Set

$$
\tilde{\theta}^{n}:=\theta 1_{\{|\theta| \leq n\}}, \quad \tau_{n}:=\inf \left\{t: \theta \cdot S_{t} \geq n\right\}, \quad \sigma_{n}:=\inf \left\{t:\left(\left(\tilde{\theta}^{n}-\theta\right) \cdot S\right)_{t}^{*} \geq 1\right\} \wedge T .
$$

Note that $\tilde{\theta}^{n} \cdot S^{\sigma_{n}} \geq a-1$. Indeed, $\tilde{\theta}^{n} \cdot S_{-}^{\sigma_{n}} \geq \theta \cdot S_{-}^{\sigma_{n}}-1$ by the definition of $\sigma_{n}$, and

$$
\Delta \tilde{\theta}^{n} \cdot S^{\sigma_{n}}=\theta 1_{\{|\theta| \leq n\}} \Delta S^{\sigma_{n}}=1_{\{|\theta| \leq n\}} \Delta \theta \cdot S^{\sigma_{n}}
$$


hence

$$
\begin{aligned}
\tilde{\theta}^{n} \cdot S^{\sigma_{n}} & =\tilde{\theta}^{n} \cdot S_{-}^{\sigma_{n}}+\Delta \tilde{\theta}^{n} \cdot S^{\sigma_{n}} \geq \theta \cdot S_{-}^{\sigma_{n}}-1+1_{\{|\theta| \leq n\}} \Delta \theta \cdot S^{\sigma_{n}} \\
& =1_{\{|\theta| \leq n\}} \theta \cdot S^{\sigma_{n}}+1_{\{|\theta|>n\}} \theta \cdot S_{-}^{\sigma_{n}}-1 \geq a-1 .
\end{aligned}
$$

Now let $\theta^{n}:=\tilde{\theta}^{n} 1_{\llbracket 0, \sigma_{n} \wedge \tau_{n} \rrbracket}$. Then $\theta^{n} \cdot S=\tilde{\theta}^{n} \cdot S^{\sigma_{n} \wedge \tau_{n}} \geq a-1$, and

$$
\begin{aligned}
\theta^{n} \cdot S & =\theta^{n} \cdot S_{-}+\Delta \theta^{n} \cdot S \leq \tilde{\theta}^{n} \cdot S_{-}^{\sigma_{n} \wedge \tau_{n}}+\theta 1_{\{|\theta| \leq n\}} \Delta S^{\sigma_{n} \wedge \tau_{n}} \\
& \leq \theta \cdot S_{-}^{\sigma_{n} \wedge \tau_{n}}+1+2 c n \leq n+1+2 c n .
\end{aligned}
$$

Hence $\theta^{n} \in \Theta_{b}$. On the other hand, we have $\left(\left(\tilde{\theta}^{n}-\theta\right) \cdot S\right)_{T}^{*}=\left(\left(\theta 1_{\{|\theta|>n\}}\right) \cdot S\right)_{T}^{*} \rightarrow 0$ in probability (note that $\theta \in L(S)$ if and only if $\left(\left(\theta 1_{\{|\theta| \leq n\}}\right) \cdot S\right)_{n \in \mathbb{N}}$ is a Cauchy sequence w.r.t. the semimartingale topology). This implies also that $P\left(\sigma_{n}<T\right) \rightarrow 0$ (i.e., $\sigma_{n} \nearrow T$, stationarily, a.s. $)$, thus $\left(\left(\theta^{n}-\tilde{\theta}^{n}\right) \cdot S\right)_{T}^{*} \rightarrow 0$ in probability. Hence $\left(\left(\theta^{n}-\theta\right) \cdot S\right)_{T}^{*} \rightarrow 0$ in probability. Finally, since $\theta^{n} \cdot S$ is uniformly bounded from below by $a-1$, and $U$ is bounded from above, we can use as above the dominated convergence theorem to conclude $\lim _{n \rightarrow \infty} E\left[U\left(\theta^{n} \cdot S_{T}+B\right)\right]=E\left[U\left(\theta \cdot S_{T}+B\right)\right]$.

Proof of Corollary 2.3. Let $\Theta_{b b} \subset \Theta \subset \Theta_{V}$. For any $\theta \in \Theta$, we have by Young's inequality,

$$
U\left(\theta \cdot S_{T}+B\right) \leq V\left(\lambda \frac{d Q}{d P}\right)+\lambda \frac{d Q}{d P}\left(\theta \cdot S_{T}+B\right), \quad \forall \lambda>0, \forall Q \in \mathcal{M}_{V},
$$

hence

$$
\begin{aligned}
E\left[U\left(\theta \cdot S_{T}+B\right)\right] & \leq E\left[V\left(\lambda \frac{d Q}{d P}\right)+\lambda \frac{d Q}{d P} B\right]+\lambda E^{Q}\left[\theta \cdot S_{T}\right] \\
& \leq E\left[V\left(\lambda \frac{d Q}{d P}\right)+\lambda \frac{d Q}{d P} B\right], \quad \forall \theta \in \Theta, \forall \lambda>0, \forall Q \in \mathcal{M}_{V},
\end{aligned}
$$

since $\theta \cdot S$ is a supermartingale under each $Q \in \mathcal{M}_{V}$. Then Theorem 2.1 implies that

$$
\begin{aligned}
\sup _{\theta \in \Theta} E\left[U\left(\theta \cdot S_{T}+B\right)\right] & \leq \inf _{\lambda>0} \inf _{Q \in \mathcal{M}_{V}} E\left[V\left(\lambda \frac{d Q}{d P}\right)+\lambda \frac{d Q}{d P} B\right] \\
& =\sup _{\theta \in \Theta_{b b}} E\left[U\left(\theta \cdot S_{T}+B\right)\right],
\end{aligned}
$$

The converse inequality follows from the inclusion $\Theta_{b b} \subset \Theta$. Finally, if $U(\infty)<\infty$, we can replace all $\Theta_{b b}$ above by $\Theta_{b}$, and the proof is complete.

\section{ACKNOWLEDGMENT}

The author warmly thanks to Prof. Koichiro Takaoka for carefully reading the manuscript, and giving a number of valuable comments. Also, the financial support from the Global Center of Excellence program "the Research Unit for Statistical and Empirical Analysis in Social Sciences" of Hitotsubashi University is gratefully acknowledged.

\section{REFERENCES}

[1] Becherer, D. (2003): Rational hedging and valuation of integrated risks under constant absolute risk aversion. Insurance Math. Econom. 33, 1-28

[2] Bellini, F., and M. Frittelli (2002): On the existence of minimax martingale measures. Math. Finance 12(1), 1-21

[3] Biagini, S., M. Frittelli, and M. Grasselli (2008): Indifference price with general semimartingales. Preprint 
[4] Cvitanić, J., W. Schachermayer, and H. Wang (2001): Utility maximization in incomplete markets with random endowment. Finance Stoch. 5(2), 259-272

[5] Delbaen, F., P. Grandits, T. Rheinländer, D. Samperi, M. Schweizer, and C. Stricker (2002): Exponential hedging and entropic penalties. Math. Finance 12(2), 99-123

[6] Frittelli, M., and E. Rosazza Gianin (2004): Equivalent formulations of reasonable asymptotic elasticity. Tech. Rep. 12, Dept. Matematica per le Decisioni, University of Florence

[7] Hugonnier, J., and D. Kramkov (2004): Optimal investment with random endowments in incomplete markets. Ann. Appl. Probab. 14(2), 845-864

[8] Hugonnier, J., D. Kramkov, and W. Schachermayer (2004): On utility based pricing of contingent claims in incomplete markets. Math. Finance 15(2), 203-212

[9] Jacod, J. (1979): Calcul Stochastique et Problèmes des Martingales, Lecture Notes in Math., vol. 714. Springer-Verlag

[10] Jacod, J. (1980): Intégrales stochastiques par rapport à une semi-martingale vectorielle et changements de filtration. In: Séminaire de Probabilités de Strasbourg XIV, Lecture Notes in Math., vol. 485, pp. 161-172. Springer-Verlag

[11] Kabanov, Y. M., and C. Stricker (2002): On the optimal portfolio for the exponential utility maximization: remarks to the six-author paper. Math. Finance 12(2), 125-134

[12] Kramkov, D., and W. Schachermayer (2003): Necessary and sufficient conditions in the problem of optimal investment in incomplete markets. Ann. Appl. Probab. 13(4), $1504-1516$

[13] Owari, K. (2008): Robust exponential hedging and indifference valuation. Discussion Paper No. 2008-9, Hitotsubashi University. http://hdl . handle.net/ $10086 / 16932$

[14] Owen, M. P., and G. Žitković (2009): Optimal investment with an unbounded random endowment and utility-based pricing. Math. Finance 19(1), 129-159

[15] Rockafellar, R. T. (1971): Integrals which are convex functionals. II. Pacific J. Math. 39(2), 439-469

[16] Rockafellar, R. T., and R. J.-B. Wets (1998): Variational Analysis, Grundlehren Math. Wiss.enchaften, vol. 317. Springer-Verlag

[17] Schachermayer, W. (2001): Optimal investment in incomplete markets when wealth may become negative. Ann. Appl. Probab. 11(3), 694-734 